УДК 616.12-008.313.3-073.97-71-08

https://doi.org/10.20538/1682-0363-2021-3-213-218

Первый опыт использования неинвазивного картирования в режиме реального времени в условиях электрофизиологической лаборатории Аля лечения желудочковой аритмии

Артюхина Е.А., Дедух Е.В., Яшков М.В., Ревишвили А.Ш.

Национальный медицинский исследовательский центр (НМИЦ) хирургии им. А.В. Вишневского Россия, 117997, г. Москва, Большая Серпуховская ул., 27

PEЗЮME

Представлен клинический случай лечения желудочковой аритмии из выводного отдела правого желудочка с использованием неинвазивного электрофизиологического картирования в режиме реального времени и отечественной навигационной системы «Астрокард» (АО «Медитек», Россия). Данное клиническое наблюдение демонстрирует точность неинвазивного картирования в режиме реального времени для диагностики и лечения желудочковых нарушений ритма сердца.

Ключевые слова: неинвазивное многоканальное картирование сердца, электрофизиологическое картирование, желудочковые аритмии, радиочастотная аблация.

Конфликт интересов. Авторы декларируют отсутствие явных и потенциальных конфликтов интересов, связанных с публикацией настоящей статьи.

Источник финансирования. Работа выполнена в рамках гранта Российского научного фонда (проект № 19-15-00406).

Для цитирования: Артюхина Е.А., Дедух Е.В., Яшков М.В., Ревишвили А.Ш. Первый опыт использования неинвазивного картирования в режиме реального времени в условиях электрофизиологической лаборатории для лечения желудочковой аритмии. Бюллетень сибирской медищины. 2021; 20 (3): 213-218. https:// doi.org/10.20538/1682-0363-2021-3-213-218.

\title{
The first experience of using non-invasive real-time mapping in an electrophysiology laboratory for treatment of ventricular arrhythmia
}

\section{Artyukhina E.A., Dedukh E.V., Yashkov M.V., Revishvili A.Sh.}

A.V. Vishnevsky National Medical Research Center of Surgery

27, Bolshaya Serpukhovskaya Str., Moscow, 117997, Russian Federation

\section{ABSTRACT}

A clinical case of treatment for ventricular arrhythmia from the right ventricular outflow tract using non-invasive real-time electrophysiological mapping and the "Astrocard" navigation system ("Meditek", Russia) is presented. This clinical case demonstrates the accuracy of non-invasive real-time mapping for the diagnosis and treatment of ventricular arrhythmias.

Дедух Елизавета Викторовна, e-mail: dedukh.elizaveta@mail.ru 
Key words: non-invasive multichannel cardiac mapping, electrophysiological mapping, ventricular arrhythmias, radiofrequency ablation.

Conflict of interest. The authors declare the absence of obvious or potential conflict of interest related to the publication of this article.

Source of financing. The study was carried out within the Russian Science Foundation grant (Project No. 19-1500406).

For citation: Artyukhina E.A., Dedukh E.V., Yashkov M.V., Revishvili A.Sh. The first experience of using noninvasive real-time mapping in an electrophysiology laboratory for treatment of ventricular arrhythmia. Bulletin of Siberian Medicine. 2021; 20 (3): 213-218. https://doi.org/10.20538/1682-0363-2021-3-213-218.

\section{ВВЕДЕНИЕ}

Радиочастотная катетерная аблация - это основной метод интервенционного лечения желудочковой экстрасистолии (ЖЭС). Она способствует улучшению качества жизни пациента и предотвращению развития кардиомиопатий и сердечной недостаточности [1]. Несмотря на то, что такие технологии, как трехмерное картирование, катетеры для аблации с мониторингом силы контакта, помогают операторам в картировании и устранении ЖЭС, эффективность и безопасность подобных операций по-прежнему зависит от локализации очага аритмии [2]. Также процесс картирования усложняет, а иногда делает невозможным возникновение «быстрых», неустойчивых и полиморфных желудочковых тахикардий. Алгоритмы диагностики с использованием электрокардиографии (ЭКГ) могут идентифицировать область происхождения желудочковых аритмий, но их пространственная локализация недостаточно точная, что объясняется различными факторами (ожирение пациента, особенности расположения сердца в грудной клетке и нарушения в размещении отведений ЭКГ) [3].

Более точным методом диагностики ЖЭС является неинвазивное картирование. Хотя эффективность неинвазивного картирования в топической диагностике ЖЭС, происходящей из выводного отдела правого желудочка (ВОПЖ), известна, и об этом сообщалось ранее $[4,5]$. Методика, используемая в данном клиническом случае, применялась впервые для диагностики желудочковых нарушений ритма сердца. Данная методика позволяет картировать правый и левый желудочки в режиме реального времени (интраоперационно) и идентифицировать по абсолютному местоположению локализацию очага экстрасистолии.

Цель данного клинического наблюдения - проверить этот метод, сравнив расчетный очаг происхождения ЖЭС на карте, полученной в результате неинвазивного картирования в режиме реального времени, и локализацию очага на системе трехмерного навигационного картирования во время катетерной аблации.

\section{КЛИНИЧЕСКИЙ СЛУЧАЙ}

Пациент А., 64 года, обратился с жалобами на ощущение перебоев в работе сердца, приступы внезапного учащенного сердцебиения, утомляемость.

Анамнез заболевания. С молодого возраста приступы внезапного учащенного сердцебиения. Во время выполнения планового медицинского осмотра выявлены желудочковые экстрасистолы на электрокардиограмме. В течение последних месяцев приступы участились, стала беспокоить повышенная утомляемость.

Дооперационное обследование. ЭКГ - синусовый ритм, частая ЖЭС с морфологией блокады левой ножки пучка Гиса (рис. 1).

Суточное мониторирование ЭКГ по Холтеру. Основной ритм синусовый, средняя частота 81 уд./ мин. Одиночные ЖЭС 28 937; бигеминия 58; парные ЖЭС 42; пробежки неустойчивой желудочковой тахикардии (ЖТ) 9. Наибольшая по продолжительности ЖТ состояла из 26 комплексов.

Эхокардиография. Правый желудочек - приносящий тракт: 28 мм. Фракция выброса (ФВ) по Симпсону $47 \%$. Толщина миокарда левого желудочка в норме. Локальных нарушений сократимости нет. Умеренная митральная и небольшая трикуспидальная регургитации. Тахиаритмия (экстрасистолия). Сбросов крови нет. Во время исследования возник приступ тахикардии с частотой сердечных сокращений 127-133, в результате чего ФВ по Симпсону снизилась до $38 \%$, увеличилась площадь митральной регургитации.

Коронароангиография. Сбалансированный тип коронарного кровоснабжения. Гемодинамически значимых стенозов коронарных артерий не выявлено.

Неинвазивное многоканальное картирование. Регистрация поверхностных электрограмм с 224 
поверхностных электродов с торса пациента, с последующим выполнением мультиспиральной компьютерной томографии (МСКТ) в режиме ЭКГ-синхронизации с контрастированием желудочков. Далее в условиях электрофизиологической лаборатории у пациента зафиксирована неустойчивая желудочковая тахикардия, данные зарегистрированы в программно-аппаратном комплексе «АМИКАРД 01К». Обработка и анализ данных происходили интраоперационно (рис. 2).

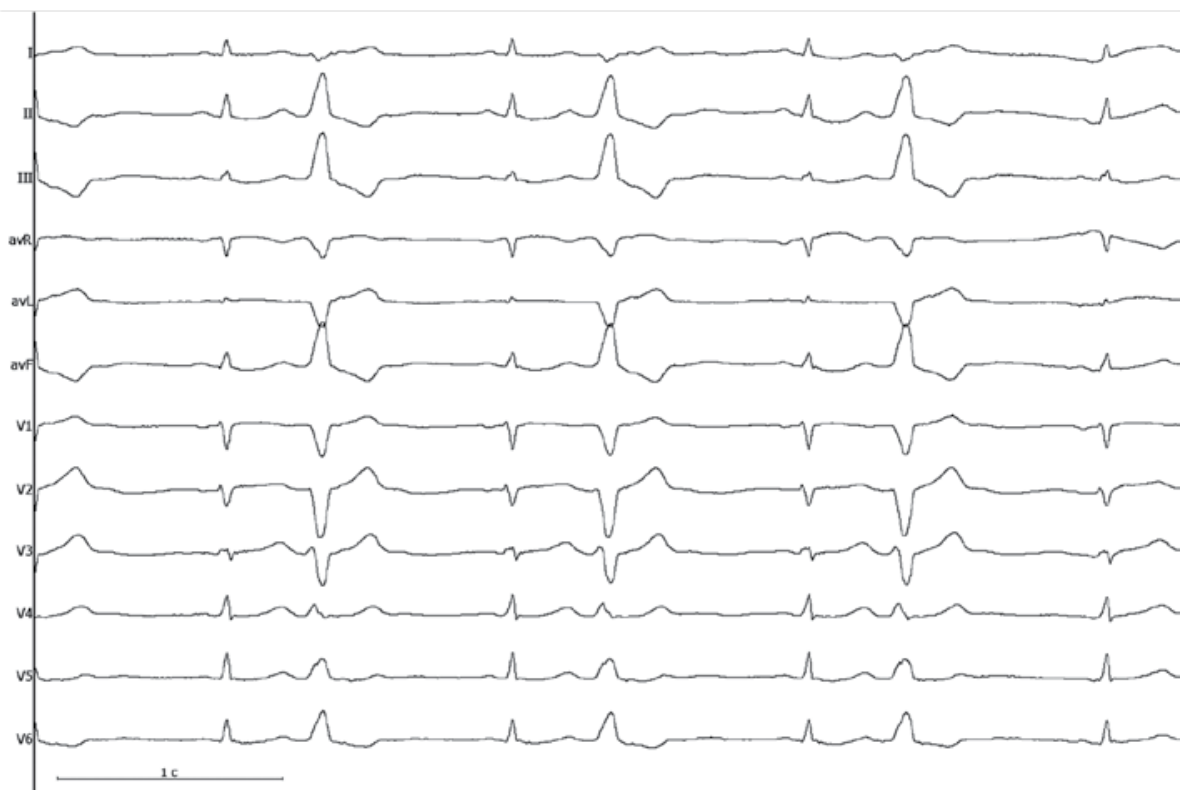

Рис. 1. Двенадцатиканальная ЭКГ, синусовый ритм с желудочковой экстрасистолией по типу бигеминии

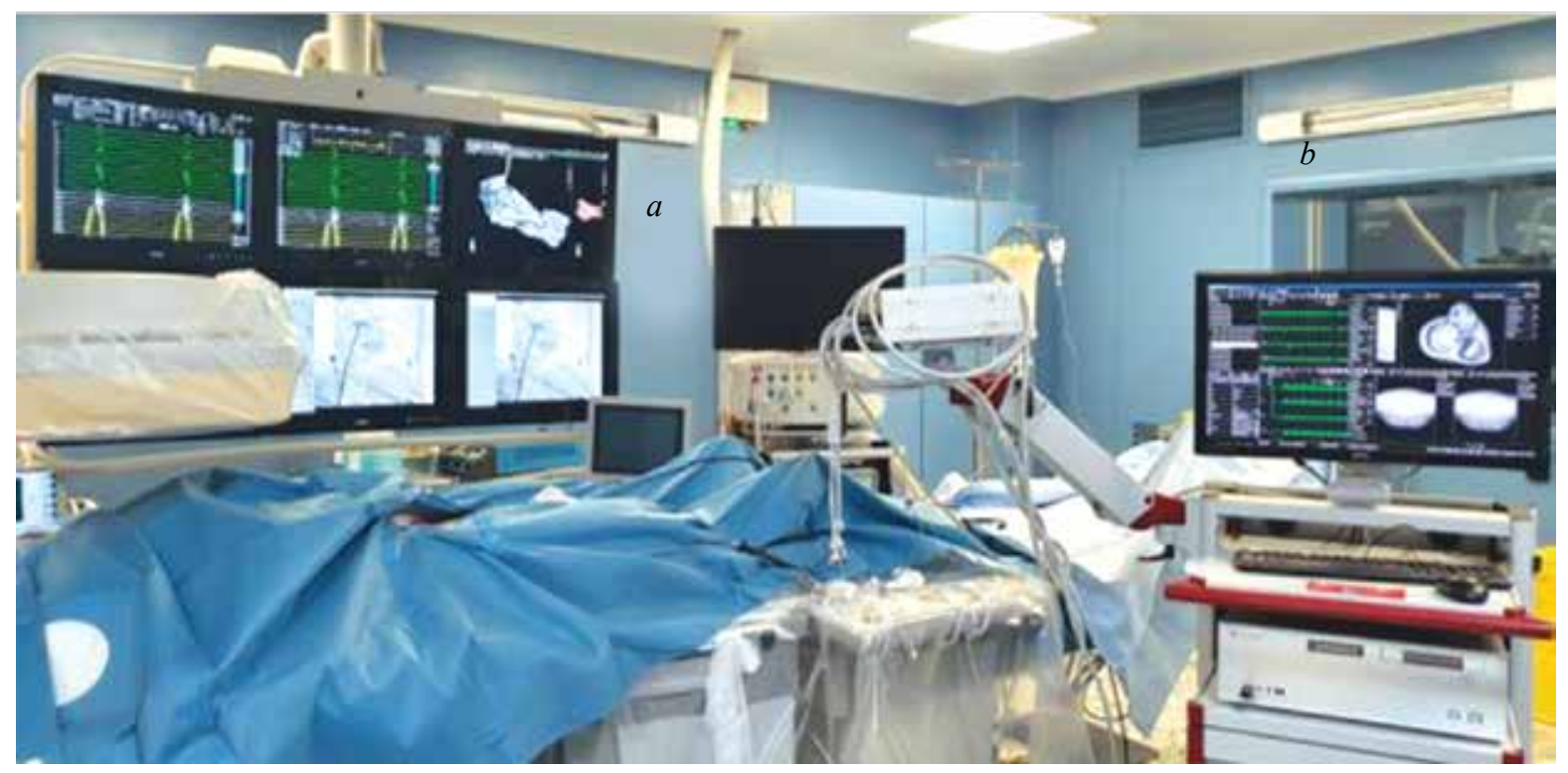

Рис. 2. Процесс картирования и лечения желудочковой экстрасистолии в условиях электрофизиологической лаборатории: $a-$ флюороскопический контроль и данные отечественной навигационной системы «Астрокард» (АО «Медитек», Россия); $b$ - программно-аппаратный комплекс «АМИКАРД 01К» (компания «Амикард», Россия)

При анализе полученной трехмерной модели желудочков сердца область ранней активации выявлена на эндокардиальной поверхности в выводном отделе правого желудочка. Результат неинвазивного картирования в режиме реального времени - изохрон- ная карта желудочков сердца (рис. 3), которая была импортирована в систему навигационного картирования. Это позволило более точно сравнивать локализацию зоны ранней активации во время желудочковой тахикардии. 


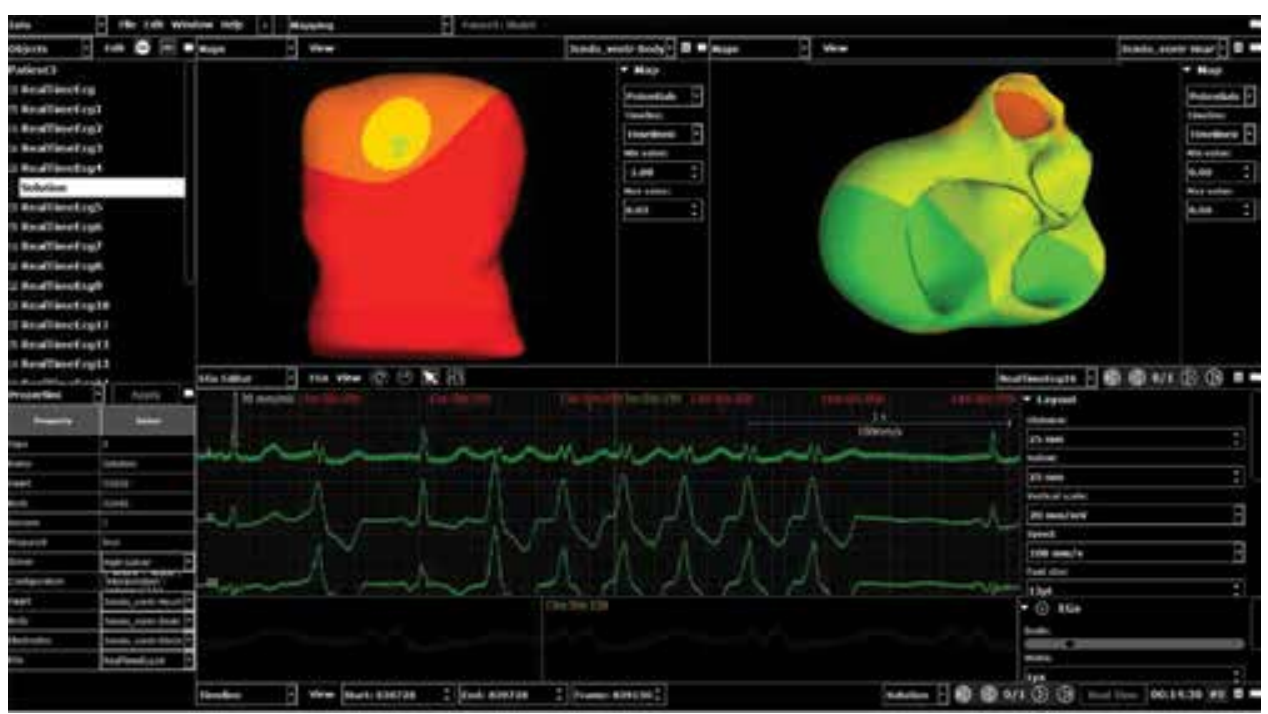

Рис. 3. Результат многоканального картирования в режиме реального времени, выполненного интраоперационно в электрофизиологической лаборатории: карта активации с ранней зоной (красный цвет) в области выводного отдела правого желудочка

\section{ИНТРАОПЕРАЦИОННОЕ КАРТИРОВАНИЕ И РАДИОЧАСТОТНАЯ АБЛАЦИЯ}

В условиях электрофизиологической лаборатории пунктированы правая бедренная и левая подключичная вены, через которую позиционирован электрод в коронарный синус. Регистрируются частая ЖЭС и эпизоды неустойчивой ЖТ с распространением электрической активности из выводного отдела правого желудочка.
Выполнена анатомическая реконструкция ПЖ c использованием отечественной навигационной системы «Астрокард», куда были импортированы данные неинвазивного картирования. Построена активационная карта правого желудочка. Данные эндокардиального картирования постоянно сопоставлялись с данными неинвазивного картирования путем визуализации правого желудочка в соответствующих проекциях (рис. 4).

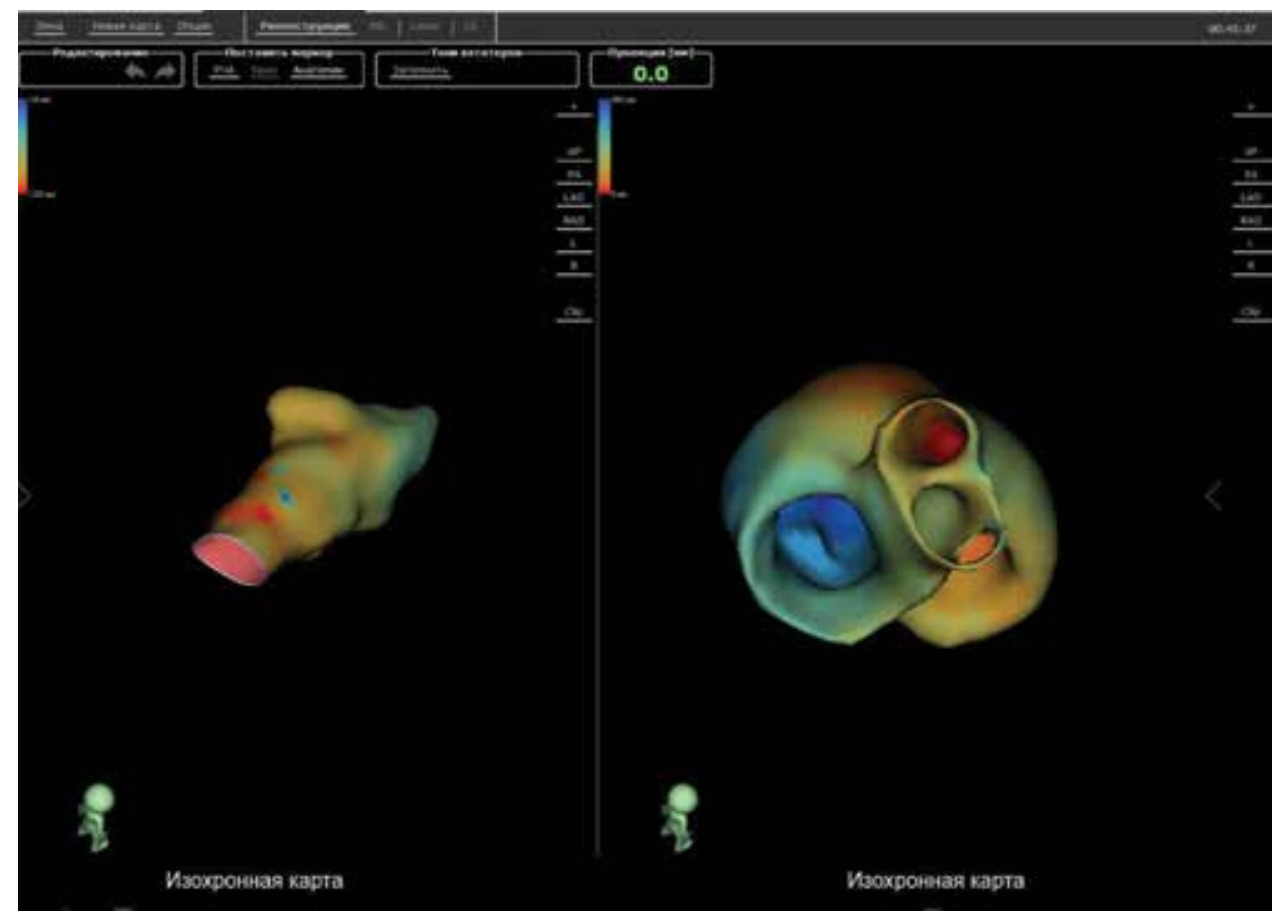

Рис. 4. Эндокардиальное картирование правого желудочка в условиях электрофизиологической лаборатории: в систему навигационного картирования импортирован результат неинвазивного картирования (справа) 
При визуализации в режиме распространения возбуждения отмечается зона ранней активации в области выводного отдела правого желудочка, что полностью совпадает с результатами неинвазивного картирования. В этой области выполнены радиочастотные воздействия, после воздействия - полная элиминация желудочковой активности.

Общая длительность воздействия составила 7 мин, параметры радиочастотного воздействия с использованием орошаемого аблационного электрода составили 35 Вт. Отсутствие экстрасистолии после воздействия - в течение 20 мин. Методами стимуляции нарушения ритма не индуцируются. Срок наблюдения составил 3 мес, нарушения ритма не регистрируются.

Несмотря на то, что данный клинический случай описывает типичную и наиболее распространенную локализацию ЖЭС, используемая методика применяется впервые. Она сможет продемонстрировать возможности картирования в режиме реального времени для диагностики желудочковых нарушений ритма сердца, что будет крайне важным шагом в картировании «быстрых» и «полиморфных» ЖТ для изучения механизма их возникновения и успешного интервенционного лечения.

\section{ОБСУЖАЕНИЕ}

Наиболее распространенными являются две системы неинвазивного картирования сердца: CardioInsight Technologies Inc., Кливленд, Огайо [6, 7] и EP Solutions SA, Yverdon-les-Bains, Швейцария [8-10].

В исследовании S. Jamil-Copley и соавт. обследованы 24 пациента с ЖЭС из выводного отдела правого желудочка (18 пациентов) и выводного отдела левого желудочка (6 пациентов) [6]. Всем пациентам, включенным в исследование, было выполнено неинвазивное картирование на дооперационном этапе. Точность определения локализации экстрасистолии, по данным неинвазивного картирования в сравнении с данными инвазивного картирования, составила 96\%.

E. Wissner и соавт. в своем исследовании показали, что у 18 (86\%) из 21 пациента с ЖЭС/ЖТ правильный желудочковый сегмент был диагностирован в результате неинвазивного картирования по единичным экстрасистолам [11].

Фактически метод неинвазивного картирования потенциально может позволить точно диагностировать эпикардиальное или эндокардиальное происхождение и камеру возникновения ЖЭС. В данном клиническом случае напрямую сравнивалась локализация ЖЭС, полученная при анализе ЭКГ и данных инвазивного эндокардиального картирования, а также данных неинвазивного картирования, полученного в режиме реального времени. В ранее про- веденных исследованиях эпикардиальные прорывы, полученные с использованием обоих методов, привели к очень большим средним абсолютным ошибкам, превышающим 70 мм [12].

На сегодняшний день электрофизиологическая природа «полиморфных» ЖТ и фибрилляции желудочков (ФЖ) все еще окончательно не выяснена. Этому препятствует гемодинамическая нестабильность пациента во время развития данных аритмий, требующая незамедлительной кардиоверсии. Основным методом изучения механизмов аритмий на сегодняшний день является эндокардиальное электроанатомическое картирование сердца. Картирование аритмии с целью изучения ее механизма возможно только при большом количестве точек, регистрирующих локальную активность сердца во время аритмии. Это возможно только при периодических и устойчивых аритмиях, при которых сохраняется стабильная гемодинамика.

ФЖ и «полиморфные» ЖТ вызывают резкое угнетение центральной гемодинамики. Они могут спонтанно прекращаться или трансформироваться в другие, более опасные аритмии. Это делает невозможным выполнение эндокардиального элетроанатомического картирования. Поэтому крайне важной задачей для изучения механизмов и интервенционного лечения жизнеугрожающих желудочковых аритмий является разработка метода, позволяющего картировать ЖТ при минимальной регистрации электрической активности миокарда во время аритмии.

Методика неинвазивного картирования в режиме реального времени, описанная в данном клиническом случае, позволит преодолеть многие из этих ограничений.

Эта методика может предоставить важную информацию о базовом механизме аритмии с использованием одного эктопического сокращения, которое будет зафиксировано в условиях электрофизиологической лаборатории, где на основании эти данных возможно выполнение интервенционного лечения желудочковой аритмии.

\section{ВЫВОДЫ}

1. Новый метод неинвазивного картирования в режиме реального времени сработал успешно и идентифицировал локализацию ЖЭС в выводном отделе правого желудочка.

2. Продемонстрирована высокая точность неинвазивного картирования в режиме реального времени в сравнении с инвазивным, что позволило эффективно устранить ЖЭС методом катетерной аблации.

3. С теоретической и математической точек зрения, эта технология имеет потенциал для определения трансмурального распределения участков 
происхождения (эпикардиальное или эндокардиальное происхождение).

Таким образом, это исследование показало только предварительные данные, но они помогут сделать возможным картирование жизнеугрожающих, «быстрых» и «полиморфных» ЖТ, которые влекут за собой нарушения стабильности гемодинамики. Метод неинвазивного картирования в режиме реального времени дополнительно усиливает преимущества системы неинвазивного картирования и расширяет клиническую применимость метода при лечении жизнеугрожающих желудочковых аритмий.

\section{ЛИТЕРАТУРА}

1. Zhong L., Lee Y.H., Huang X.M., Asirvatham S.J, Shen W.K., Friedman P.A., Hodge D.O., Slusser J.P., Song Z.Y., Packer D.L., Cha Y.M. Relative efficacy of catheter ablation vs antiarrhythmic drugs in treating premature ventricular contractions: a single-center retrospective study. Heart Rhythm. 2014; 11 (2):187-193. DOI: 10.1016/j.hrthm.2013.10.033.

2. Komatsu Y., Nogami A., Shinoda Y, Masuda K., Machino T., Kuroki K., Yamasaki H., Sekiguchi Y., Aonuma K. Idiopathic ventricular arrhythmias originating from the vicinity of the communicating vein of cardiac venous systems at the left ventricular summit. Circ. Arrhythm. Electrophysiol. 2018; 11 (1): e005386. DOI: 10.1161/CIRCEP.117.005386.

3. Anter E., Frankel D.S., Marchlinski F.E., Dixit S. Effect of electrocardiographic lead placement on localization of outflow tract tachycardias. Heart Rhythm. 2012; 9 (5): 697-703. DOI: 10.1016/j.hrthm.2011.12.007.

4. Inaba T., Nakazawa Y., Yoshida K., Kato Y., Hattori A., Kimura T., Hoshi T., Ishizu T., Seo Y., Sato A., Sekiguchi Y., Nogami A., Watanabe S., Horigome H., Kawakami Y., Aonuma K. Routine clinical heart examinations using SQUID magnetocardiography at university of tsukuba hospital. Supercond. Sci. Technol. 2017; 30 (11). DOI: 10.1088/1361-6668/aa8c26.

5. Ito Y., Shiga K., Yoshida K., Ogata K., Kandori A., Inaba T., Nakazawa Y., Sekiguchi Y., Tada H., Sekihara K., Aonuma K. Development of a magnetocardiography-based algorithm for discrimination between ventricular arrhythmias originating from the right ventricular outflow tract and those originating from the aortic sinus cusp: a pilot study. Heart Rhythm. 2014; 11 (9): 1605-1612. DOI: 10.1016/j.hrthm.2014.05.032.
6. Jamil-Copley S., Bokan R., Kojodjojo P., Qureshi N., KoaWing M., Hayat S., Kyriacou A., Sandler B., Sohaib A., Wright I., Davies D.W., Whinnett Z., Peters S.N., Kanagaratnam P., Lim P.B. Noninvasive electrocardiographic mapping to guide ablation of outflow tract ventricular arrhythmias. Heart Rhythm. 2014; 11 (4): 587-594. DOI: 10.1016/j.hrthm.2014.01.013.

7. Cakulev I., Sahadevan J., Arruda M., Goldstein R.N., Hong M., Intini A., Mackall J.A., Stambler B.S., Ramanathan C., Jia P., Strom M., Waldo A.L. Confirmation of novel noninvasive high-density electrocardiographic mapping with electrophysiology study: implications for therapy. Circ. Arrhythm. Electrophysiol. 2013; 6 (1): 68-75. DOI: 10.1161/CIRCEP.112.975813.

8. Revishvili A.S. , Wissner E., Lebedev D.S., Lemes C., Deiss S., Metzner A., Kalinin V.V., Sopov O.V., Labartkava E.Z., Kalinin A.V., Chmelevsky M., Zubarev S.V., Chaykovskaya M.K., Tsiklauri M.G., Kuck K.H. Validation of the mapping accuracy of a novel non-invasive epicardial and endocardial electrophysiology system. Europace. 2015; 17 (8): 1282-1288. DOI: 10.1093/europace/euu339.

9. Wissner E., Saguner A.M., Metzner A., Chmelesky M., Tsyganov A., Deiss S., Maurer T., Kuck K.H. Radiofrequency ablation of premature ventricular contractions originating from the aortomitral continuity localized by use of a novel noninvasive epicardial and endocardial electrophysiology system. Heart Rhythm Case Rep. 2016; 2 (3): 255-257. DOI: 10.1016/j. hrcr.2016.02.004.

10. Wang Y., Cuculich P.S., Zhang J., Desouza K.A., Vijayakumar R., Chen J., Faddis M.N., Lindsay B.D., Smith T.W., Rudy Y. Noninvasive electroanatomic mapping of human ventricular arrhythmias with electrocardiographic imaging. Sci. Transl. Med. 2011; 3 (98): 98ra84. DOI: 10.1126/scitranslmed.3002152.

11. Wissner E., Revishvili A., Metzner A., Tsyganov A., Kalinin V., Lemes C., Saguner A.M., Maurer T., Deiss S., Sopov O., Labarkava E., Chmelevsky M., Kuck K.H. Noninvasive epicardial and endocardial mapping of premature ventricular contractions. Europace. 2017; 19 (5): 843-849. DOI: 10.1093/ europace/euw103.

12. Duchateau J., Sacher F., Pambrun T., Derval N., Chamorro-Servent J., Denis A., Ploux S., Hocini M., Jaïs P., Bernus O., Haïssaguerre M., Dubois R. Performance and limitations of noninvasive cardiac activation mapping. Heart Rhythm. 2019; 16 (3): 435-442. DOI: 10.1016/j.hrthm.2018.10.010.

\section{Сведения об авторах}

Артюхина Елена Александровна, д-р мед. наук, руководитель отделения электрофизиологических рентгенэндоваскулярных методов диагностики и лечения аритмий, НМИЦ хирургии им. А.В. Вишневского, г. Москва. ORCID 0000-0001-7065-0250.

Дедух Елизавета Викторовна, аспирант, НМИЦ хирургии им. А.В. Вишневского, г. Москва. ORCID 0000-0002-4799-7456.

Яшков Максим Валерьевич, аспирант, НМИЦ хирургии им. А.В. Вишневского, г. Москва. ORCID 0000-0003-3997-8252.

Ревишвили Амиран Шотаевич, д-р мед. наук, профессор, академик РАН, директор НМИЦ хирургии им. А.В. Вишневского, г. Москва. ORCID 0000-0003-1791-9163.

(凹) Дедух Елизавета Викторовна, e-mail: dedukh.elizaveta@mail.ru 